\title{
Macrolide antibiotic exposure in the first trimester of pregnancy and risk of congenital anomaly: A European case-control study
}

\author{
Aminkeng Leke ${ }^{1}$, Helen Dolk ${ }^{2}$, Maria Loane ${ }^{3}$, Karen Casson ${ }^{1}$, Vera Nelen ${ }^{4}$, Ingeborg \\ Barisic $^{5}$, Ester Garne ${ }^{6}$, Anke Rissmann ${ }^{7}$, Mary O’Mahony ${ }^{8}$, Amanda Neville ${ }^{9}$, Anna \\ Pierini $^{10}$, Jorieke Bergman ${ }^{11}$, Kari Klungsoyr ${ }^{12}$, Anna Materna-Kiryluk ${ }^{13}$, Anna \\ Bielenska $^{13}$, Clara Cavero-Carbonell ${ }^{14}$, Marie-Claire Addor ${ }^{15}$, and David Tucker ${ }^{16}$ \\ ${ }^{1}$ Ulster University - Jordanstown Campus \\ ${ }^{2}$ University of Ulster School of Nursing \\ ${ }^{3}$ Ulster University \\ ${ }^{4}$ Provinciaal Instituut voor Hygiene \\ ${ }^{5}$ Medical School University of Zagreb \\ ${ }^{6}$ Paediatric Department Hospital \\ ${ }^{7}$ Otto von Guericke University Medical Faculty \\ 8 St Finbarr's Hospital \\ ${ }^{9}$ University of Ferrara Faculty of Medicine and Surgery \\ ${ }^{10}$ National Research Council / Fondazione Toscana Gabriele Monasterio \\ ${ }^{11}$ University of Groningen, University Medical Center Groningen \\ ${ }^{12}$ University of Bergen \\ ${ }^{13}$ Poznan University of Medical Sciences \\ ${ }^{14}$ Foundation for the Promotion of Health and Biomedical Research in the Valencian Region \\ ${ }^{15}$ University Hospital of Lausanne \\ ${ }^{16} \mathrm{NHS}$
}

September 10, 2020

\begin{abstract}
Objective To investigate the risk of congenital heart defects (CHD) and other congenital anomalies (CA) associated with first trimester use of macrolides. Design Population-based case-malformed control study. Setting Thirteen European countries. Population Data on 145,936 livebirths, stillbirths and terminations of pregnancy for CA from 15 EUROCAT registries, covering 9 million births 1995-2012. Methods Cases were babies with CHD, anencephaly, orofacial clefts, genital and limb reduction anomalies associated with antibiotic exposure in the literature. Controls were babies with other CA or genetic conditions. A meta-analysis of the literature, including this study, was conducted for CHD. Main outcome Odds ratios adjusted (AOR) for maternal age and registry, with 95\% Confidence Intervals (95\% CI). Results Macrolide exposure was recorded for 307 cases, 72 non-genetic controls, 57 genetic controls. AOR for CHD was not significantly raised (AOR 0.94, 95\%CI: $0.70-1.26$ vs non-genetic controls; AOR 1.01, 95\%CI: 0.73 - 1.41 vs genetic controls), nor significantly raised for any specific macrolide. The risk of atrioventricular septal defect was significantly raised with exposure to any macrolide (AOR 2.98; 95\%CI: $1.48-$ 6.01), erythromycin (AOR 3.68, 95\%CI: 1.28 - 10.61), and azithromycin (AOR 4.50, 95\%CI: 1.30 - 15.58). Erythromycin, clarithromycin, azithromycin and clindamycin, were also associated with an increased risk of at least one other CA. Metaanalysis gave an overall CHD OR 1.14, 95\% CI 0.90 - 1.49 for macrolides. Conclusions Guidelines for macrolide use in pregnancy should consider the increased risk of specific CA. This is relevant for the potential use of azithromycin in the treatment of
\end{abstract}


COVID-19.

Macrolide antibiotic exposure in the first trimester of pregnancy and risk of congenital anomaly: A European case-control study

Aminkeng Zawuo Leke ${ }^{1} ;$ Helen Dolk $^{1}$; Maria Loane ${ }^{1}$; Karen Casson ${ }^{1}$; Vera Nelen ${ }^{2}$; Ingeborg Barišíc ${ }^{3}$; Ester Garne $^{4}$; Anke Rissman ${ }^{5}$; Mary O'Mahony ${ }^{6}$; Amanda J Neville ${ }^{7}$; Anna Pierini ${ }^{8}$; Jorieke E.H. Bergman ${ }^{9}$; Kari Klungsøyr ${ }^{10}$; Anna Materna-Kiryluk ${ }^{11}$; Anna Latos Bielenska ${ }^{12}$; Clara Cavero Carbonell ${ }^{13}$; Marie-Claude Addor ${ }^{14}$; David Tucker ${ }^{15}$

1. Centre for Maternal, Fetal and Infant Research, Institute for Nursing and Health Research, Ulster University-UK

2. Provinciaal Instituut voor Hygiëne, Antwerp, Belgium

3. Children's Hospital Zagreb, Centre of Excellence for Reproductive and Regenerative Medicine, Medical School University of Zagreb, Zagreb, Croatia, Zagreb, Croatia

4. Paediatric Department Hospital, Lillebaelt Skovvangen, Kolding, Denmark

5. Malformation Monitoring Centre Saxony-Anhalt, Medical Faculty Otto-von-Guericke University Magdeburg, Germany

6. Medicine Department of Public Health, St Finbarr's Hospital Douglas Road, Cork, Ireland

7. IMER Registry (Emila Romagna Registry of Birth Defects), Center for Clinical and Epidemiological Research, University of Ferrara - Azienda Ospedaliero - Universitaria di Ferrara, Corso della Giovecca, Ferrara, Italy

8. Tuscany Registry of Congenital Defects, CNR Institute of Clinical Physiology/Fondazione Toscana Gabriele Monasterio, Pisa, Italy

9. Department of Medical Genetics, University of Groningen, University Medical Center Groningen, Groningen, The Netherlands

10. Medical Birth Registry of Norway, Kalfarveien, Bergen, Norway

11. Polish Registry of Congenital Malformations, Department of Medical Genetics, Poznan University of Medical Sciences, Poznan, Poland

12. Poznan University of Medical Sciences, Department of Medical Genetics. 8 Rokietnicka Street, 60-806 Poznan, Poland.

13. Rare Diseases Research Unit, Foundation for the Promotion of Health and Biomedical Research in the Valencian Region, Valencia, Spain.

14. Registre Vaudois des Malformations EUROCAT Department of Woman-Mother-Child, Maternité, Lausanne, Switzerland

15. Congenital Anomaly Register \& Information Service, Level 3 West Wing, Singleton Hospital, Sketty Lane, Swansea, United Kingdom

Corresponding Author: Aminkeng Zawuo Leke

Centre for Maternal, Fetal and Infant Research, Institute for Nursing and Health Research,

Ulster University, Shore Road. Newtownabbey, Co. Antrim

BT37 0QB, Northern Ireland United Kingdom

Email: az.leke@ulster.ac.uk; Tel: +4407568214655

Running title: Macrolides use in pregnancy and risk of congenital anomalies

Tweetable abstract: Macrolide antibiotics, including azithromycin considered for COVID-19 treatment, are associated with increased risk of birth defects.

The likelihood of getting LS by age 80 years is

$1.6 \%$. The mortality of women with LS is reduced compared with 
The likelihood of getting LS by age 80 years is

$1.6 \%$. The mortality of women with LS is reduced compared with

\section{Abstract \\ Objective}

To investigate the risk of congenital heart defects (CHD) and other congenital anomalies (CA) associated with first trimester use of macrolides.

\section{Design}

Population-based case-malformed control study.

\section{Setting}

Thirteen European countries.

\section{Population}

Data on 145,936 livebirths, stillbirths and terminations of pregnancy for CA from 15 EUROCAT registries, covering 9 million births 1995-2012.

\section{Methods}

Cases were babies with CHD, anencephaly, orofacial clefts, genital and limb reduction anomalies associated with antibiotic exposure in the literature. Controls were babies with other CA or genetic conditions. A meta-analysis of the literature, including this study, was conducted for CHD.

\section{Main outcome}

Odds ratios adjusted (AOR) for maternal age and registry, with 95\% Confidence Intervals (95\%CI).

\section{Results}

Macrolide exposure was recorded for 307 cases, 72 non-genetic controls, 57 genetic controls. AOR for CHD was not significantly raised (AOR 0.94, 95\%CI: $0.70-1.26$ vs non-genetic controls; AOR 1.01, 95\%CI: $0.73-$ 1.41 vs genetic controls), nor significantly raised for any specific macrolide. The risk of atrioventricular septal defect was significantly raised with exposure to any macrolide (AOR 2.98; 95\%CI: $1.48-6.01$ ), erythromycin (AOR 3.68, 95\%CI: 1.28 - 10.61), and azithromycin (AOR 4.50, 95\%CI: 1.30 - 15.58). Erythromycin, clarithromycin, azithromycin and clindamycin, were also associated with an increased risk of at least one other CA. Meta-analysis gave an overall CHD OR 1.14, 95\%CI $0.90-1.49$ for macrolides.

\section{Conclusions}

Guidelines for macrolide use in pregnancy should consider the increased risk of specific CA. This is relevant for the potential use of azithromycin in the treatment of COVID-19.

\section{Funding}

Study conducted as part of a $\mathrm{PhD}$ funded by Ulster University.

Keywords: Macrolides, Erythromycin, Spiramycin, Clarithromycin, Azithromycin, Clindamycin, Pregnancy, First trimester, Congenital anomaly

\section{Introduction}

Macrolide antibiotics are commonly used to treat respiratory problems and certain sexually transmitted diseases. ${ }^{1-3}$ They are generally considered to have a good safety profile, but there have been conflicting findings about their potential to prolong the QT interval in ECG tests of the heart. ${ }^{1-4}$ Azithromycin, an analogue of erythromycin, is considered to have a better safety profile than erythromycin and clarithromycin. ${ }^{1,4}$ It has a slow-acting anti-malarial property; and owing to growing resistance to sulphadoxine-pyrimethamine 
(SP), its combination with chloroquine has been proposed as an alternative to the current WHO recommended intermittent preventive treatment of malaria in pregnancy with SP. ${ }^{5-7}$ Currently, the potential use of azithromycin - chloroquine combination in COVID -19 treatment is being tested. ${ }^{8}$

Macrolides are widely used during pregnancy, but evidence of their teratogenic potential is limited and inconsistent. Animal studies have generally found limited evidence of teratogenicity of macrolides. However, ever since the thalidomide disaster in the 1960s, animal studies are not considered adequate to predict risk in humans. ${ }^{9}$ Pregnant women are excluded from human trials to determine drug efficacy and safety for ethical reasons. ${ }^{9}$ Safety information needed by pregnant women and their clinicians to guide risk and benefit analysis must, therefore, come from post-marketing pharmaco-epidemiological studies.

There has been a growing debate about the potential association of macrolides (especially erythromycin) with congenital heart defects (CHD). In 2003, a Swedish Birth Registry study reported that first trimester use of macrolides (mainly erythromycin) was associated with increased risk for CHD (OR 1.79; 95\% CI 1.3 2.8). ${ }^{10}$ Subsequent updates of this study in 2005 and 2013 found similar results. ${ }^{11,12}$ A recent UK study has also corroborated this result, reporting an increased risk of CHD (adjusted relative risk 1.62; 95\% CI 1.05 2.51). ${ }^{13}$ Other studies have found no significant association between macrolides and $\mathrm{CHD}^{14-28}$, including a 2019 meta-analysis. ${ }^{29}$ Most studies did not have an adequate sample size and power to investigate specific CHD. Most teratogens are specific to the defects they cause ${ }^{30}$ and analysing congenital anomalies (CA) in large groups may result in missed associations of the exposure with specific defects.

We investigate here the teratogenic potential of five macrolide antibiotics, as part of a wider study of antibiotics using the large European EUROmediCAT database (http://www.euromedicat.eu/currentresearchanddata/data), derived from registries belonging to the EUROCAT network for Surveillance of Congenital Anomalies (https://eu-rdplatform.jrc.ec.europa.eu/eurocat/eurocat-network_en). We also conduct a meta-analysis of the literature for CHD, including our own results.

\section{Methods}

\section{Study design}

The EUROmediCAT database only holds data for babies with CA among live births (LB), fetal deaths (FD) from 20 weeks gestational age and terminations of pregnancy following prenatal diagnosis of fetal anomaly (TOPFA) at any gestational age. It does not include any data on babies without CA that may be used as non-malformed controls. We therefore performed a case-malformed control study. Cases were babies with CA associated in the epidemiologic literature with any antibiotic exposure. Controls were babies with other CA or genetic conditions. To avoid confusion, throughout this paper the term 'registration' refers to babies/fetuses with CA including both cases and controls

\section{Study population and data}

EUROmediCAT is a network dedicated to the study of medication safety in pregnancy which includes EUROCAT population-based CA registries collecting data on medication exposure during pregnancy. Currently 20 EUROCAT registries from 14 countries participate in EUROmediCAT with annual surveillance covering approximately 753,000 births per year throughout Europe (www.euromedicat.eu) ${ }^{32}$.

The registries collect and send anonymised individual case data to the EUROmediCAT Central database. The standard data on each registration are described in EUROCAT Guide 1.4. ${ }^{31}$ One syndrome and up to eight malformations are coded using International Classification of Diseases, Ninth Revision (ICD-9) or Tenth Revision (ICD-10) codes with British Pediatric Association (BPA)/ $\mathrm{RCPCH}$ one-digit extensions. Registrations with only anomalies on the EUROCAT list of minor anomalies are excluded leaving registrations with one or more major malformations. This list of minor anomalies includes also anomalies of non-congenital origin including pyloric stenosis, and Patent Ductus Arteriosus in preterm births. All registrations are classified to EUROCAT subgroups according to their ICD9/10-RCPCH codes, as set out in EUROCAT Guide 1.4. ${ }^{1}$ The sources used to obtain data on CA vary across registries and include maternity, neonatal, and paediatric 
records; fetal medicine, cytogenetic, pathology, and medical genetics records; paediatric cardiology services; and hospital discharge and child health records. ${ }^{33}$ The majority of the registries record anomalies diagnosed up to at least one year of age.

Medications taken in the first trimester of pregnancy are coded according to the Anatomic Therapeutic Chemical (ATC) classification. Maternal disease before and during pregnancy are recorded using ICD codes. Most registries (84\%) collect prospective data on medication exposure during the first trimester, mainly from maternity and other medical files, and some collect retrospective data from interviews with women after delivery or other sources, either alone $(17 \%)$ or in combination with prospective sources $(28 \%)^{34}$. All participating registries reviewed antibiotic exposures to confirm that they were first trimester exposures.

All registries recording information on antibiotic exposures (regardless of timing), with data over the period of this study (1995-2012), were eligible to participate in the study. Eighteen of the 23 (at start of study) eligible registries agreed to participate in the study and gave permission for their data to be extracted.

\section{Dataset exclusions}

We applied the following exclusions to our original dataset:

1) the South East (SE) Ireland registry was excluded from the dataset because there were no registrations with a first trimester antibiotic exposure; Reunion-France and Ukraine registries were excluded because they recorded no macrolide exposure;

2) TOPFAs were excluded from the Emilia Romagna registry as information on medications is only available for LB and stillbirths;

3) registrations with isolated hip dislocation/dysplasia were excluded based on the known association with birthweight and potential for confounding;

4) registrations with mothers with epilepsy or pregestational diabetes, or mothers who took anti-epileptics or hypoglycaemic agents were excluded due to the known association with CA;

5) registrations which had antibiotic exposures of unknown timing or missing description were excluded (90\% of these were from three registries: Saxony-Anhalt-Germany, Wielkopolska-Poland and Poland excluding Wielkopolska). These exclusions reduced the dataset from 170,062 to 145,936 registrations as shown in Figure 1.

\section{Case definition}

Cases were defined as all LB, FD from 20 weeks gestational age, and TOPFA with CA types (corresponding to EUROCAT subgroups) which were signals identified from the literature, excluding those with a genetic syndrome (see Figure 2 and supplementary Table S1). We conducted a literature review of case-control and cohort studies reported in Medline and Reprotox databases from creation up to March 2016, to identify signals of specific CA associated with first trimester use of antibiotics. We defined a signal as a statistically significant result of increased risk, or a risk ratio [?] 3, with at least two exposed cases in the first trimester. ${ }^{35}$ As this report focuses on macrolides, the signals identified for the macrolides up to 2016 are shown in Table 1.

\section{Control definition}

We used two control groups:

Non-genetic controls: LB, FD from 20 weeks gestation, and TOPFA with all other major CA subgroups, excluding CA subgroup types that were defined as cases or related to case CA subgroups, and excluding genetic syndromes (Figure 2).

Genetic controls: LB, FD from 20 weeks' gestation, and TOPFA, with a diagnosis of a genetic syndrome (Figure 2). 


\section{Exposures under investigation and exposure comparison groups}

Exposure in first trimester of pregnancy to any macrolide (ATC code J01FA), and five specific macrolide subclass erythromycin (ATC code J01FA01), spiramycin (ATC code J01FA02), clarithromycin (ATC code J01FA09), azithromycin (ATC code J01FA10) and clindamycin (ATC code J01FF01)). Two types of exposure comparison groups were used: no antibiotic exposure during pregnancy (primary comparison) and exposure to drugs other than antibiotics, excluding vitamins and minerals (secondary comparison).

Macrolides are used as an alternative for pregnant women who are allergic to penicillins which is considered relatively safer compared to other types of antibiotics. ${ }^{13}$ Hence we performed sensitivity analyses for selected significant results using penicillins as the exposure comparison in order to account for any residual confounding due to infection. This approach has also been used in other studies. ${ }^{13,19,25}$

\section{Statistical Methods}

Data were analysed using STATA version 12. Odds ratios (OR), crude (COR) and adjusted (AOR), were produced by logistic regression. We performed analysis for macrolides as a class, then for each of the five subclasses of macrolides. Registries without any exposures for a specific macrolide subclass were excluded from the analysis of that subgroup. Each investigation of an association between a macrolide and a case CA subgroup made use of the four comparison groups i.e. the two control groups (non-genetic controls and genetic controls) and the two exposure comparison groups i.e. no antibiotics exposure (primary exposure comparison) and exposed to other medications (secondary exposure comparison). We adjusted for the following confounders: year of birth in three time periods (1995 - 2000; 2001 - 2006; 2007 - 2012), EUROCAT registry, and maternal age in categories $(<20 ; 20-24 ; 25-29 ; 30-34 ; 35-39$ and $40+)$.

We divided our analyses into signal testing analyses regarding the five subgroups of CA shown in Table 1, and exploratory analyses regarding the non-macrolide antibiotic signal CA subgroups. For the latter, all results are given in supplementary files, and only statistically significant results with at least 3 exposed cases are given in the main tables.

We considered a result to be robust if AOR were statistically significant in two or more control and exposure comparison groups. Only results involving the primary exposure comparison group are presented (results involving secondary exposure comparison are found in the Supplementary Tables S4b-9b).

As our primary controls (non-genetic controls) are malformed babies, there is a possibility that some CA in this control group could be associated with the macrolides under investigation (teratogen non-specificity bias), leading to reduced OR. ${ }^{36,37}$ As a sensitivity analysis, we therefore investigated the association between nongenetic control anomalies and macrolides using the secondary controls (genetic controls) as the comparison group. These results are given in the Supplementary Table S3.

\section{Meta-analysis}

In the light of the debate in the literature regarding the association between macrolides and CHD, we performed a meta-analysis using RevMan 5.3. We updated the literature review described above to February 2020. For all macrolides (J01FA), we combined our results with that of a recent meta-analysis of CHD and macrolides ${ }^{29}$ and one new study ${ }^{13}$ not included in the published meta-analysis. We took into consideration the heterogeneity result from the recent CHD-macrolide meta-analysis and also investigated heterogeneity among studies included in our meta-analysis. Assuming OR provide an unbiased estimate of relative risk in rare outcomes as $\mathrm{CA},{ }^{38}$ we combined case-control and cohort studies to obtained pooled OR. Statistical significance level was set at 0[?]05 and results were reported as OR and 95\% CI.

\section{Results}

Our analysis dataset consisted of 145,936 registrations from 15 EUROCAT registries in 13 European countries covering an 18-year period (1995 to 2012) and included 100,702 cases, 23,467 non-genetic controls and 19,060 genetic controls (Table 2). A total of 2,707 registrations with CA subgroups related to that of cases were excluded from the analysis (Figure 2). 
During the first trimester, a total of $3,440(2.36 \%)$ women were exposed to at least one antibiotic (See Supplementary Table S2). First trimester infection was reported for 2,499 (1.71\%) women of whom only $14.77 \%$ had a record of first trimester antibiotic exposure.

Cases significantly differed from both control groups with respect to registry and maternal age (Table 2). Cases and controls also differed by type of birth, since the proportion of TOPFA varies by type of anomaly.

\section{Signal testing and exploratory analysis}

Overall, the proportion of cases $(0.30 \%)$ exposed to macrolides in the first trimester was not significantly different to that of non-genetic controls $(0.29 \%$; AOR 0.99; 95\%CI 0.76-1.28), or genetic controls $(0.28 \%$; AOR 1.04; 95\%CI 0.77-1.40). Similar results were also obtained for all five specific macrolides studied (Table $3)$.

None of the 5 signals shown in Table 1 were confirmed (Table 3). However, although the CHD signal was not confirmed, a pattern of increased risk for the specific heart defect atrioventricular septal defect (AVSD) was observed for any macrolide exposure (9 exposed cases, AOR 2.98; 95\%CI 1.48-6.01); erythromycin (4 exposed cases, AOR 3.68; 95\%CI 1.28-10.61); clarithromycin (2 exposed cases, AOR 6.85; 95\%CI 1.41-33.32; see foot notes in Table 4); and azithromycin (3 exposed cases, AOR 4.50; 95\%CI 1.30-15.58) (Table 4). These associations were robust across both control and exposure comparison groups (see also Supplementary Tables S4, S5, S7 and S8).

Since the 2016 literature review generating the signal CA, one further study has generated new signals: urinary system with all macrolides and respiratory system with moxifloxacin. ${ }^{25}$ These CA subgroups were both included among non-genetic controls. There was no evidence of any increased risk for these CA subgroups compared to genetic controls: urinary system (AOR $0.8795 \%$ CI $0.55-1.39$ ); respiratory system (AOR 0.44; 95\%CI 0.10-1.89) (Supplementary Table S3).

In the exploratory analyses of signal CA subgroups previously associated with other antibiotics, four associations were found (Table 3): erythromycin with diaphragmatic hernia (5 exposed cases, AOR 3.19; 95\% CI 1.22-8.32); clarithromycin with orofacial clefts (8 exposed cases, AOR 2.94; 95\%CI 1.04-8.30); azithromycin with syndactyly (8 exposed cases, AOR 3.80; 95\%CI 1.62-8.94); and clindamycin with hydrocephalus (3 exposed cases, AOR 6.63; 95\% CI 1.46, 30.18).

\section{Sensitivity analysis}

Sensitivity analyses for all significantly elevated risks using penicillins as the exposure comparison group, gave AOR similar or greater than that obtained for no antibiotics (primary exposure comparison), although with less precision (Supplementary Table S10). The study results were also not altered when we excluded the three registries with a high proportion of excluded registrations due to unknown antibiotic timing from the analyses.

The overall risk for non-genetic controls as a group was not raised compared to genetic controls (AOR 1.01; 95\%CI 0.69-1.46), confirming absence of strong macrolide associations in this control group. With regard to specific CA in the non-genetic control group, we found an increased risk with macrolide exposure for teratogenic syndromes with malformations (AOR 6.50; 95\% CI 1.92-22.03) explained by its subcategory maternal infections resulting in malformations (OR 6.44; 95\%CI 1.89-21.92) (Supplementary Table S3). These are recognised infection syndromes such as congenital rubella/CMV/toxoplasmosis, where antibiotic use may be expected, but numbers are very small. Results from further analysis after excluding these teratogenic syndromes from the controls were similar to the original study results.

The study results were similar when the analyses were restricted to case subgroups with isolated CA, and when genetic controls with signal anomalies were excluded.

\section{Meta-analysis}

Apart from two recent studies conducted in $2019^{26}$ and $2020^{13}$, all the studies included in the meta-analysis 
had already been evaluated and reported in the meta-analysis of Mallah et al., 2019. ${ }^{29}$ The latter found there was no heterogeneity among the studies included in their meta-analysis. In our study, we observed heterogeneity in the macrolide meta-analysis, but not for the macrolide subclasses. Results of the pooled estimates, including the findings for the current study, did not show a significant increased risk of CHD following first trimester use of macrolides, erythromycin, clarithromycin or azithromycin (Figure $3 \mathrm{~A}-\mathrm{D}$ ).

\section{Discussion}

In this large EUROmediCAT study, we did not find evidence of a strong association between CHD and macrolide exposure, and meta-analysis of our study combined with all previous literature confirmed an overall lack of a strong association. However, we did find evidence regarding a threefold or more raised risk of AVSD specifically, significantly associated with three types of macrolide, robust across analyses with different control groups and exposure comparison groups. AVSD accounts for $2 \%$ of CHD cases in EUROCAT data, so it is not surprising this does not affect the overall CHD finding. AVSD are common in babies with Down syndrome, but none of the exposed AVSD cases had Down syndrome. The majority of negative studies regarding macrolides and CHD,,$^{14-26}$ as well as those that found an association, ${ }^{10}-13$ did not have enough power to investigate specific subgroups of CHD. We found only one study (Crideret al. 2009) ${ }^{17}$ that has investigated the association between AVSD and erythromycin and found an elevated risk, although not statistically significant (AOR 2.2, CI: $0.8-6.1$ ). This was a case-control study which obtained exposure information retrospectively by interview a considerable time after exposure, thus possibly underestimating ORs.

As elaborated by Kallen et al . ${ }^{11}$ there is compelling evidence from animal and human studies that suggests macrolides could have a link with some CHDs. At clinical concentrations, macrolides can inhibit a specific cardiac potassium current (IKr) channel, expressed by hERG (human ether a-go-go related gene). This can then lead to a prolonged QT interval, causing a type of ventricular tachycardia called torsades de pointes (TdP). ${ }^{4,39}$ In a developing rat embryo, particularly during the period before the heart is inverted (corresponding to weeks 5 - 9 of human pregnancy), TdP can result in pressure changes and misdirection of blood flow in the developing cardiovascular system, that can in turn lead to hypoxia and re-oxygenation damage resulting in septal and other vessel defects. ${ }^{41-44}$

Determining the cause of birth defects is complex, as a single birth defect may have multiple causes, just as multiple birth defects can have a single cause. However, our current AVSD finding has met many of the widely accepted Bradford Hill criteria of causation, ${ }^{45}$ including the large effect size, observed pattern specific to macrolides, existence of prior hypothesis regarding CHD and a plausible biological mechanism.

In other exploratory analysis, we have found robust elevated risk of diaphragmatic hernia, orofacial clefts, syndactyly, and hydrocephalus, associated with first trimester use of erythromycin, clarithromycin, azithromycin, and clindamycin respectively. None of these associations have been previously reported. However, multiple testing may have produced some spuriously significant results, and independent confirmation is necessary.

We could not confirm previous associations of macrolides with genital anomalies, ${ }^{21}$ erythromycin with anencephaly ${ }^{17}$ or limb deficiency, ${ }^{17}$ and azithromycin with orofacial clefts. ${ }^{16}$ Since our 2016 literature search, a new study found an association of urinary system defects with erythromycin (AOR 2.12, CI: $1.08-4.17$ ), ${ }^{25}$ which our data and other studies did not support. ${ }^{12,21,46-48}$

Our investigation of spiramycin did not find an association with any of the CAs studied, but this was the least frequent exposure and we had limited statistical power. We found only one study from the literature that investigated spiramycin, finding no increased risk. ${ }^{27}$ Further studies are needed on the teratogenic potential of this antibiotic.

Apart from CA, other adverse outcomes related to macrolide use during pregnancy have been reported, such as miscarriage ${ }^{49}$ and fetal growth restriction in animal studies of clarithromycin and azithromycin. ${ }^{50-52}$

Safety advice about the use of macrolides during pregnancy varies across different countries. In the United Kingdom, the Medicines and Healthcare products Regulatory Agency advises alternatives to clarithromycin 
and azithromycin should be prescribed during pregnancy. ${ }^{53}$ In Sweden, the report of Kallen et al . ${ }^{11}$ in 2005 that suggested elevated risk of CHD related to erythromycin led to warning against its use in the first trimester. ${ }^{53}$ In the USA, the previous Food and Drug Agency pregnancy classification system classified erythromycin, azithromycin and clindamycin as category B (animal studies found no risk and there are no adequately controlled human studies), and clarithromycin as category $\mathrm{C}$ (animal studies show adverse effects, but there are no adequately controlled human studies and benefit may warrant use despite potential risks). In light of the findings from the current study, regulatory authorities should revise the safety advice on macrolide use during the first trimester of pregnancy to reflect the potential risk of adverse outcomes. As the world considers the potential use of azithromycin - chloroquine combination in the treatment of COVID $-19{ }^{8}$ particular attention should be given to the potential teratogenic and other negative effects of azithromycin use during the first trimester of pregnancy.

\section{Strengths and limitations}

The major strengths of our study include the population based data, large sample size, detailed and standardised data on CA, and inclusion of TOPFA, which constitute a large proportion of some CAs. ${ }^{55}$ Additionally, exposures were mainly prospectively ascertained, and our case-malformed control design limits recall and information bias, especially for the small proportion of retrospectively ascertained exposures. Our study was hypothesis driven, and the use of two control and exposure comparison groups together with sensitivity analysis, allowed us to evaluate the robustness of any associations.

Our study also had some limitations. Exposure to antibiotics (2.36\%) was low, compared with the expected $3-14 \%$ rate of first trimester antibiotic exposure in the European population. ${ }^{10,19,56}$ This suggests antibiotic exposures were under-ascertained in our data, as was shown also in a study comparing the registry data to linked prescription data. ${ }^{57}$ The reporting of antibiotic exposures would not, however, have been different between cases and controls and thus should not lead to biased odds ratios. We had very little data about the indication for prescribing, or about untreated infection, so we could not examine confounding by indication in this way. Our sensitivity analysis comparing macrolide use with penicillins obtained similar results to that with the primary exposure comparison. Since macrolides and penicillins are commonly used for the same indication, this suggests that the excess risk we found relates to macrolides rather than the underlying infection. Other studies have used this same approach as a proxy to account for residual confounding by indication. ${ }^{12,19,25}$ We examined evidence of teratogen non-specificity bias and found our results to be robust; they were similar after excluding two control subgroups which were associated with antibiotics, and similar for the secondary comparison with genetic syndromes which cannot be associated with first trimester medication exposure. Finally, we conducted many tests with some significant findings possible by chance, and, therefore, specified our hypotheses in advance, and recommend confirmation of new (exploratory) findings in independent datasets.

\section{Conclusion}

Our investigation of five CA signals related to macrolides found negative results. While a positive association was not found between CHD as a group and macrolides, an elevated risk was found between macrolides and specific macrolides (erythromycin, clarithromycin and azithromycin) and AVSD. We also found elevated risk for other defects associated with first trimester use of specific macrolides that need further follow up in independent datasets. Guidelines on the teratogenic risk of macrolide use during the first trimester of pregnancy should take the current findings into account. These results should also guide recommendations for pregnant women when azithromycin is considered as part of COVID-19 treatment.

\section{Acknowledgments}

We are grateful to Ulster University whose Vice Chancellor PhD Research Scholarship to LAZ made this project possible. We are also grateful to the French West Indies Registry for contributing data to this study; and to the SE Ireland, Ukraine and Reunion-France registries which contributed data, although these did not meet the inclusion criteria for this study. 


\section{Disclosure of interests}

We declare no competing interests.

\section{Contribution to authorship}

AZL, HD, ML and KC conceptualised the study. VN, IB, EG, HR, AR, MO, AJN, AP,JEHB, KK, AMK, $\mathrm{ALB}, \mathrm{CCC}, \mathrm{MCA}$, DT extracted data and verified all first trimester exposures. LAZ conducted the literature review and data analysis with technical inputs from HD, ML and KC. AZL, HD drafted the manuscript with full inputs from all other authors.

\section{Ethical approval}

This study was approved by the INHR Ethics Committee in Ulster University on the $11^{\text {th }}$ of March 2012 . All contributing registries have their own ethics approval arrangements appropriate to their national and local ethics guidelines.

Funding: AZL received the Ulster University Vice Chancellor PhD Research Scholarship (2013-2017).

\section{References}

1. Giudicessi J, Ackerman M. Azithromycin and risk of sudden cardiac death: Guilty as charged or falsely accused? Cleveland Clinic Journal of Medicine . 2013;80(9):539-544.

2. Gorelik E, Masarwa R, Perlman A, Rotshild V, Muszkat M, Matok I. Systematic Review, Metaanalysis, and Network Meta-analysis of the Cardiovascular Safety of Macrolides. Antimicrobial Agents and Chemotherapy. 2018;62(6).

3. Polgreen L, Riedle B, Cavanaugh J, Girotra S, London B, Schroeder M et al. Estimated Cardiac Risk Associated With Macrolides and Fluoroquinolones Decreases Substantially When Adjusting for Patient Characteristics and Comorbidities. Journal of the American Heart Association . 2018;7(9).

4. Juurlink D. The cardiovascular safety of azithromycin. Canadian Medical Association Journal . 2014;186(15):1127-1128.

5. Chico R, Pittrof R, Greenwood B, Chandramohan D. Azithromycin-chloroquine and the intermittent preventive treatment of malaria in pregnancy. Malaria Journal . 2008;7(1).

6. WHO policy brief for the implementation of intermittent preventive treatment of malaria in pregnancy using sulfadoxine-pyrimethamine (IPTp-SP) [Internet]. World Health Organization. 2014 [cited 1 June 2020]. Available from: https://www.who.int/malaria/publications/atoz/policy_brief_iptp_sp_policy_recommendation/en/

7. Kimani J, Phiri K, Kamiza S, Duparc S, Ayoub A, Rojo R et al. Efficacy and Safety of AzithromycinChloroquine versus Sulfadoxine-Pyrimethamine for Intermittent Preventive Treatment of Plasmodium falciparum Malaria Infection in Pregnant Women in Africa: An Open-Label, Randomized Trial. PLOS ONE . 2016;11(6):e0157045.

8. Timothy F. Simpson; Richard J. Kovacs; Eric C. Stecker. Ventricular Arrhythmia Risk Due to Hydroxychloroquine-Azithromycin Treatment For COVID-19 - American College of Cardiology [Internet]. American College of Cardiology. 2020 [cited 1 June 2020]. Available from: https://www.acc.org/latest-in-cardiology/articles/2020/03/27/14/00/ventricular-arrhythmiarisk-due-to-hydroxychloroquine-azithromycin-treatment-for-covid-19

9. Ridings JE. The thalidomide disaster, lessons from the past. InTeratogenicity Testing. 2013; 575-586. Humana Press, Totowa, NJ.

10. Kallen B. Maternal drug use in early pregnancy and infant cardiovascular defect. Reproductive Toxicology . 2003;17(3):255-261.

11. Kallen B, Otterblad Olausson P, Danielsson B. Is erythromycin therapy teratogenic in humans?. Reproductive Toxicology . 2005;20(2):209-214.

12. Kallen B, Danielsson B. Fetal safety of erythromycin. An update of Swedish data. European Journal of Clinical Pharmacology. 2013;70(3):355-360. 
13. Fan H, Gilbert R, O'Callaghan F, Li L. Associations between macrolide antibiotics prescribing during pregnancy and adverse child outcomes in the UK: population based cohort study. BMJ . 2020;m331.

14. Tellem R, Shechtman S, Arnon J. Pregnancy outcome after gestational exposure to the new macrolides: a prospective controlled cohort study.Reproductive Toxicology . 2005; 20: 484-485.

15. Bar-Oz B, Diav-Citrin O, Shechtman S, Tellem R, Arnon J, Francetic I et al. Pregnancy outcome after gestational exposure to the new macrolides: A prospective multi-center observational study.European Journal of Obstetrics \& Gynecology and Reproductive Biology . 2008;141(1):31-34.

16. Cooper WO, Hernandez-Diaz S, Arbogast PG, Dudley JA, Dyer SM, Gideon PS, Hall KS, Kaltenbach LA, Ray WA. Antibiotics potentially used in response to bioterrorism and the risk of major congenital malformations. Paediatric and perinatal epidemiology . 2009;23(1):18-28.

17. Crider KS, Cleves MA, Reefhuis J, Berry RJ, Hobbs CA, Hu DJ. Antibacterial medication use during pregnancy and risk of birth defects: National Birth Defects Prevention Study. Archives of pediatrics Es adolescent medicine. 2009;163(11):978-85.

18. Nordeng H, Romoren M, Lindbaek M. Safety of macrolides during pregnancy-With special focus on erythromycin and congenital heart malformations. Reproductive Toxicology . 2010;2(30):227.

19. Romoren M, Lindbaek M, Nordeng H. Pregnancy outcome after gestational exposure to erythromycina population-based register study from Norway. British journal of clinical pharmacology . 2012;74(6):1053-62.

20. Bar-Oz B, Weber-Schoendorfer C, Berlin M, Clementi M, Di Gianantonio E, de Vries L, De Santis M, Merlob P, Stahl B, Eleftheriou G, Maňáková E. The outcomes of pregnancy in women exposed to the new macrolides in the first trimester. Drug safety . 2012;35(7):589-98.

21. Lin KJ, Mitchell AA, Yau WP, Louik C, Hernández-Díaz S. Safety of macrolides during pregnancy. American journal of obstetrics and gynecology . 2013;208(3):221-e1.

22. Andersen JT, Petersen M, Jimenez-Solem E, Rasmussen JN, Andersen NL, Afzal S, Broedbaek K, Hjelvang BR, Køber L, Torp-Pedersen C, Poulsen HE. Trimethoprim use prior to pregnancy and the risk of congenital malformation: a register-based nationwide cohort study.Obstetrics and gynecology international . 2013;2013.

23. Dinur AB, Koren G, Matok I, Wiznitzer A, Uziel E, Gorodischer R, Levy A. Fetal safety of macrolides. Antimicrobial agents and chemotherapy . 2013;57(7):3307-11.

24. Bérard A, Sheehy O, Zhao JP, Nordeng H. Use of macrolides during pregnancy and the risk of birth defects: a population-based study.Pharmacoepidemiology and drug safety . 2015;24(12):1241-8.

25. Muanda FT, Sheehy O, Berard A. Use of antibiotics during pregnancy and the risk of major congenital malformations: a population based cohort study. British journal of clinical pharmacology . 2017;83(11):2557-71.

26. Damkier P, Bronniche LM, Korch-Frandsen JF, Broe A. In utero exposure to antibiotics and risk of congenital malformations: a population-based study. American journal of obstetrics and gynecology . 2019;221(6):648-e1.

27. Czeizel AE, Rockenbauer M, Olsen J, SORENSEN HT. A case-control teratological study of spiramycin, roxithromycin, oleandomycin and josamycin. Acta obstetricia et gynecologica Scandinavica . 2000;79(3):234-7.

28. Czeizel AE, Rockenbauer M, Sorensen HT, Olsen J. A population-based case-control teratologic study of oral erythromycin treatment during pregnancy. Reproductive Toxicology . 1999;13(6):531-6.

29. Mallah N, Tohidinik HR, Etminan M, Figueiras A, Takkouche B. Prenatal Exposure to Macrolides and Risk of Congenital Malformations: A Meta-Analysis. Drug safety . 2019;12:1-1.

30. Khoury MJ, Moore CA, James LM, Cordero JF. The interaction between dysmorphology and epidemiology: methodologic issues of lumping and splitting. Teratology . 1992;45(2):133-8.

31. EUROCAT guide 1.4 reference documents[Internet]. 2018 [cited 1 June 2020]. Available from: https://eu-rd-platform.jrc.ec.europa.eu/sites/default/files/Full_Guide_1_4_version_28_DEC2018.pdf

32. de Jong-van den Berg LT, Bakker MK, Dolk H. EUROmediCAT: European surveillance of safety of medication use in pregnancy.Pharmacoepidemiology and Drug Safety . 2011;20(S1):S46-7. 
33. Greenlees R, Neville A, Addor MC, Amar E, Arriola L, Bakker M, Barisic I, Boyd PA, Calzolari E, Doray B, Draper E. Paper 6: EUROCAT member registries: organization and activities. Birth Defects Research Part A: Clinical and Molecular Teratology. 2011;91(S1):S51-100.

34. Bakker M, De Jonge L. EUROCAT Special Report: Sources of information on medication use in pregnancy. EUROCAT Central Registry . 2014.

35. Leke AZ. Maternal use and safety of anti-infectives in pregnancy, with special reference to Cameroon (Doctoral dissertation, Ulster University). 2017; Chapter 4, p159.

36. Rothman KJ, Greenland S, Lash TL, editors. Modern epidemiology.Lippincott Williams $\&$ Wilkins . 2008.

37. Hook EB. What kind of controls to use in case control studies of malformed infants: recall bias versus "teratogen nonspecificity" bias. Teratology . 2000;61(5):325-6.

38. Poletta FA, Camelo JS, Gili JA, Leoncini E, Castilla EE, Mastroiacovo P. Methodological approaches to evaluate teratogenic risk using birth defect registries: advantages and disadvantages. PloS one . $2012 ; 7(10)$.

39. Webster R, Leishman D, Walker D. Towards a drug concentration effect relationship for QT prolongation and torsades de pointes. Current opinion in drug discovery $\&$ development . 2002;5(1):116-26.

40. Danielsson C, Brask J, Skold AC, Genead R, Andersson A, Andersson U, Stockling K, Pehrson R, Grinnemo KH, Salari S, Hellmold H. Exploration of human, rat, and rabbit embryonic cardiomyocytes suggests K-channel block as a common teratogenic mechanism. Cardiovascular research . 2013;97(1):23-32.

41. Davies MP, An RH, Doevendans P, Kubalak S, Chien KR, Kass RS. Developmental changes in ionic channel activity in the embryonic murine heart. Circulation research . 1996;78(1):15-25.

42. Wang L, Feng ZP, Kondo CS, Sheldon RS, Duff HJ. Developmental changes in the delayed rectifier $\mathrm{K}+$ channels in mouse heart. Circulation research . 1996;79(1):79-85.

43. Abrahamsson C, Palmer M, Ljung B, Duker G, Baarnhielm C, Carlsson L, Danielsson B. Induction of rhythm abnormalities in the fetal rat heart. A tentative mechanism for the embryotoxic effect of the class III antiarrhythmic agent almokalant. Cardiovascular research . 1994;28(3):337-44.

44. Jaffee OC. Hemodynamic factors in the development of the chick embryo heart. The Anatomical Record . 1965;151(1):69-75.

45. Hill AB. The environment and disease: association or causation?.Proc R Soc Med. 1965;58: 295-300.

46. Louik C, Werler MM, Mitchell AA. Erythromycin use during pregnancy in relation to pyloric stenosis. American journal of obstetrics and gynecology . 2002;186(2):288-90.

47. Lund M, Pasternak B, Davidsen RB, Feenstra B, Krogh C, Diaz LJ, Wohlfahrt J, Melbye M. Use of macrolides in mother and child and risk of infantile hypertrophic pyloric stenosis: nationwide cohort study.Bmj . 2014;348:g1908.

48. Cooper WO, Ray WA, Griffin MR. Prenatal prescription of macrolide antibiotics and infantile hypertrophic pyloric stenosis. Obstetrics \& Gynecology . 2002 Jul 1;100(1):101-6.

49. Fan H, Li L, Wijlaars L, Gilbert RE. Associations between use of macrolide antibiotics during pregnancy and adverse child outcomes: A systematic review and meta-analysis. PloS one . 2019;14(2).

50. Briggs GG, Freeman RK, Yaffe SJ. Drugs in pregnancy and lactation: a reference guide to fetal and neonatal risk. Lippincott Williams \& Wilkins . 2012.

51. Karabulut AK, Uysal II, Acar H, Fazliogullari Z. Investigation of developmental toxicity and teratogenicity of macrolide antibiotics in cultured rat embryos. Anatomia, histologia, embryologia. 2008 Oct;37(5):369-75.

52. Medicines \& Healthcare Products Regulatory Agency. Summary of Product Characteristics: Clarithromycin 500mg film-coated tablets. 2019 [cited 1 June 2020]. Available from: http://www.hpra.ie/img/uploaded/swedocuments/Licence_PA1347-068-002_13112019125315.pdf.

53. Joint Formulary Committee. BNF 77 (British National Formulary) March 2019. Pharmaceutical Press; 2019.

54. The Swedish Medical Products Agency. [Erythromycin should be avoided during early pregnancy]. 2006. [cited 1 June 2020]. https://lakemedelsverket.se/Alla-nyheter/NYHETER-2005/Erytromycinbor-undvikas-under-tidiggraviditet/. 
55. Garne E, Dolk H, Loane M, Eurocat PA. EUROCAT website data on prenatal detection rates of congenital anomalies.2010.

56. Petersen I, Gilbert R, Evans S, Ridolfi A, Nazareth I. Oral antibiotic prescribing during pregnancy in primary care: UK population-based study. Journal of antimicrobial chemotherapy.2010;65(10):223846.

57. de Jonge L, Garne E, Gini R, Jordan SE, Klungsoyr K, Loane M, Neville AJ, Pierini A, Puccini A, Thayer DS, Tucker D. Improving information on maternal medication use by linking prescription data to congenital anomaly registers: a EUROmediCAT study. Drug safety . 2015;38(11):1083-93.

\section{Hosted file}

Tables and Figures.docx available at https://authorea.com/users/355550/articles/478729macrolide-antibiotic-exposure-in-the-first-trimester-of-pregnancy-and-risk-of-

congenital-anomaly-a-european-case-control-study 